\title{
Effect of Different Levels of Inorganics and Biofertilizers on Post Harvest Soil Physico-Chemical Properties of Baby Corn (Zea mays L.) Variety- G-5414
}

\author{
Cici Maria Antony*, Narendra Swaroop, Tarence Thomas, \\ Arun A. David and P. Smriti Rao
}

Sam Higginbottom University of Agriculture Technology and Sciences, Department of Soil Science and Agricultural Chemistry, Prayagraj, Uttar Pradesh, 211007, India

*Corresponding author

Keywords

Baby corn, Fertility, Biofertilizers, Seed inoculation

\section{Article Info}

Accepted: 12 August 2020 Available Online: 10 September 2020

\section{A B S T R A C T}

A field experiment was conducted at crop research farm, Department of soil science and Agricultural chemistry, Sam Higginbottom University of Agriculture Technology And Sciences, Prayagraj, (Uttar Pradesh) during kharif 2019 on sandy loam soil. The experiment was laid out according to randomized block design with three replications. The experiment comprised of three fertility levels (control, 50\% recommended dose of fertilizers, $75 \%$ recommended dose of fertilizers) and three biofertiliizers levels (Azospirillum, Phosphorous solubilising bacteria, Azospirillum + Phosphorous solubilising bacteria) were applied to the baby corn variety G-5414. The application of $75 \%$ recommended dose of fertilizers and seed inoculation with Azospirillum + Phosphorous solubilising bacteria significantly increased the organic carbon, available nitrogen and potassium content in soil. However, bulk density and particle density was found non significant after harvesting of the crop because these properties don't change in one cropping season. While there was a slight increase in solid space and specific gravity in $\mathrm{T}_{10}$ (Phosphorous solubilising bacteria+Azospirillum@20 $\mathrm{g} \mathrm{kg}^{-1}+75 \%$ recommended dose of fertilizers), \%pore space and water retaining capacity was highest in $\mathrm{T}_{4}$ (Azospirillum + Phosphorous solubilising bacteria @ $20 \mathrm{~g} \mathrm{~kg}^{-1}+0 \%$ recommended dose of fertilizers).

\section{Introduction}

Maize (Zea mays L.), the Queen of cereals, is considered as one of the most important cereal crops in the world. It serves as a staple food than any of the other cereal crops. Maize (Zea mays L.) ranks $3^{\text {rd }}$ as a food-grain crop after wheat and rice and it is not only a cereal but is also used as a vegetable and fodder crop. Baby corn is an immature dehusked, unfertilized maize ear, harvested 1-2 days after silking at 2-3 cm-long silk stage and consumed as vegetables owing to its sweet flavour (Pandey et al., 1998).

The term 'Biofertilizer' in India specifies fertilizers to meet the nutritional requirements of a crop through microbiological means, 
other countries use the term 'microbial inoculants'. These biofertilizers are usually carrier based microbial preparations containing beneficial microorganisms in a viable state intended for seed or soil application, which enhance plant growth through nutrient uptake and growth hormone production (Brahmaprakash and Saha, 2004).

Azospirillum used in maize and can satisfy $20-25 \%$ of nitrogen requirement (Raj and Gaur,1982) whereas Phosphobacteria, a phosphate solubilising microorganism will make available of the phosphate sources from the un or less available form (Datta et al., 1992).

In the 1960s, with the onset of the Green Revolution (adoption of high yielding cereal varieties, chemical inputs and mechanization), agricultural systems in developing countries have achieved remarkable improvements. In developing countries such as India, more than 70 percent of the population is indulged in agriculture and allied activities. Increased productivity in turn has fed millions who otherwise would have starved out of hunger. Increase in production had been augmented with increased use of fertilizers and other agrochemicals which were a boon and a bane at the same time. The excessive use of agrochemicals (fertilizers and pesticides) is posing serious threats to the environment. Increased use of nitrogenous fertilizers delivered visible results and higher net returns but also have high adverse effects on the soil and its fertility. . Also there is evidence of stagnation or low productivity of maize and other kharif cereals even with the application of recommended doses of NPK fertilizers.

Advances in understanding of soil microbiology and biotechnology have made possible exploitation of soil microorganisms for improving crop productivity and, in turn, have offered an economically attractive and ecologically viable supplement to reduce external inputs to some extent (Khalid et al., 2009).

\section{Materials and Methods}

\section{Experimental sites}

The experiment was conducted at the research farm of department of Soil Science and Agricultural chemistry, Sam Higginbottom University of Agriculture, Technology and Sciences, Prayagraj which is situated six km away from Prayagraj city on the right bank of Yamuna river. The experimental site is located in the sub - tropical region with $25^{\circ} \mathrm{N}$ latitude $81.50^{\circ} \mathrm{E}$ longitude and $98 \mathrm{~m}$ above mean sea level. Soil samples were taken from $0-15 \mathrm{~cm}$ depth of soil randomly prior to tillage operations, airdried, and passed through $2 \mathrm{~mm}$ sieve. Then the composite sample was taken for mechanical and chemical analysis. The values of pre sowing analysis are given in Table 1.

\section{Design and treatment}

The experiment was carried out in randomized block design with three levels of N P K, three levels of Biofertilizers. The treatments were replicated three times and were allocated at random in each replication.

\section{Fertilizer application}

The recommended dose of fertilizer for baby corn (150:60:40) was supplied through urea, DAP (Di-Ammonium Phosphate) and MOP (Muriate of Potash). The nitrogen required was supplied in split a dose (15DAS and 30DAS) while DAP and MOP was provided as basal doses. Bio fertilizers were applied through seed inoculation with Azospirillum and PSB. The fertilizers were applied in each plot according to treatment combinations. $\mathrm{T}_{0^{--}}$ Control (No Treatment), T2-Azospirillum@20 
$\mathrm{gm} \mathrm{kg}^{-1}+0 \%$ RDF, T 3 PSB@20 $\mathrm{gm} \mathrm{kg}^{-1}+$ $0 \% \mathrm{RDF}, \mathrm{T}_{4-}$ Azospirillum + PSB@20 $\mathrm{gm} \mathrm{kg}^{-1}$ + 0\%RDF, T5-Azospirillum@20 gm kg-1 $50 \%$ RDF[ N(75 kg ha $\left.{ }^{-1}\right), \mathrm{P}\left(30 \mathrm{~kg} \mathrm{ha}^{-1}\right), \mathrm{K}(20$ $\left.\left.\mathrm{kg} \mathrm{ha}{ }^{-1}\right)\right], \mathrm{T}_{6-}$ PSB@20 gm kg-1 + 50\%RDF[ $\left.\mathrm{N}\left(75 \mathrm{kgha}^{1}\right), \mathrm{P}\left(30 \mathrm{~kg} \mathrm{ha}^{-1}\right), \mathrm{K}\left(20 \mathrm{~kg} \mathrm{ha}^{-1}\right)\right], \mathrm{T}_{7-}$ Azospirillum + PSB@20 gm kg $\mathrm{gm}^{-1}$ 50\%RD [ $\left.\mathrm{N}\left(75 \mathrm{~kg} \mathrm{ha}^{-1}\right), \mathrm{P}\left(30 \mathrm{~kg} \mathrm{ha}^{-1}\right), \mathrm{K}\left(20 \mathrm{~kg} \mathrm{ha}^{-1}\right)\right]$, $\mathrm{T}_{8-}$ Azospirillum@20 $\mathrm{gm} \mathrm{kg}^{-1}+75 \% \mathrm{RDF}[\mathrm{N}$ (112.5 kg ha $\left.\left.{ }^{-1}\right), \mathrm{P}\left(45 \mathrm{~kg} \mathrm{ha}^{-1}\right), \mathrm{K}\left(30 \mathrm{~kg} \mathrm{ha}^{-1}\right)\right]$, $\mathrm{T}_{9-} \mathrm{PSB} @ 20 \mathrm{gm} \mathrm{kg}^{-1}+75 \% \mathrm{RDF}[\mathrm{N}(112.5$ $\left.\left.\mathrm{kg} \mathrm{ha}{ }^{-1}\right), \mathrm{P}\left(45 \mathrm{~kg} \mathrm{ha}^{-1}\right), \mathrm{K}\left(30 \mathrm{~kg} \mathrm{ha}^{-1}\right)\right], \mathrm{T}_{10-}$ Azospirillum + PSB @ $20 \mathrm{gm} \mathrm{kg}^{-1}+$ 75\%RDF[N(112.5 $\left.\mathrm{kg} \mathrm{ha}^{-1}\right), \mathrm{P}\left(45 \mathrm{~kg} \mathrm{ha}^{-1}\right)$, $\left.\mathrm{K}\left(30 \mathrm{~kg} \mathrm{ha}^{-1}\right)\right]$.

\section{Results and Discussion}

\section{Physical properties}

\section{Bulk density $\left(\mathrm{Mg} \mathrm{m}^{-3}\right)$}

Treatment $\mathrm{T}_{10}$ (PSB+Azospirillum @ $20 \mathrm{gm}$ $\mathrm{kg}^{-1}+75 \% \mathrm{RDF} \quad\left[\mathrm{N}\left(112.5 \mathrm{~kg} \quad \mathrm{ha}^{-1}\right), \quad \mathrm{P}(45\right.$ $\left.\left.\mathrm{kg} \mathrm{ha}^{-1}\right), \mathrm{K}\left(30 \mathrm{~kg} \mathrm{ha}^{-1}\right)\right]$ recorded maximum Bulk density (1.23 $\mathrm{Mgm}^{-3}$ ) along with (1.23 $\mathrm{Mgm}^{-3}$ ) $\mathrm{T}_{8}$ (Azospirillum @ $20 \mathrm{gm} \mathrm{kg}^{-1}+$ 75\%RDF [ (112.5 kg ha-1), P(45 kg ha $\left.{ }^{-1}\right)$, $\left.\mathrm{K}\left(30 \mathrm{~kg} \mathrm{ha}^{-1}\right)\right] . \mathrm{T}_{1}$ recorded the minimum bulk density $\left(1.11 \mathrm{Mgm}^{-3}\right)$.

Physical properties do not change significantly in one cropping season. This might be reason why no significant variation in bulk density, particle density and porosity occurred with different fertility and biofertilizers levels. Similar results were found by Jangir et al., (2017).

\section{Particle density $\left(\mathrm{Mg} \mathrm{m}^{-3}\right)$}

Treatment $\mathrm{T}_{8}$ (PSB+Azospirillum@20 gm kg-1 +75\%RDF [N(112.5 kg ha $\left.{ }^{-1}\right), \mathrm{P}\left(45 \mathrm{~kg} \mathrm{ha}^{-1}\right)$, $\left.\mathrm{K}\left(30 \mathrm{~kg} \mathrm{ha}^{-1}\right)\right]$ recorded maximum Particle density (2.96 $\left.\mathrm{Mgm}^{-3}\right)$ followed by (2.92 $\mathrm{Mgm}^{-3}$ ) $\mathrm{T}_{10}$ (Azospirillum + PSB@20 gm kg-1
+75\%RDF[ N(112.5 $\left.\mathrm{kg} \mathrm{ha}^{-1}\right), \mathrm{P}\left(45 \mathrm{~kg} \mathrm{ha}^{-1}\right)$, $\left.\mathrm{K}\left(30 \mathrm{~kg} \mathrm{ha} \mathrm{ha}^{-1}\right)\right] . \mathrm{T}_{1}$ recorded the minimum particle density $\left(2.5 \mathrm{Mgm}^{-3}\right)$. Physical properties do not change significantly in one cropping season. Similar results were found by Jangir et al., (2017).

\section{$\%$ pore space}

Treatment $\mathrm{T}_{4}$ (Azospirillum+PSB@20g kg-1 + $0 \% \mathrm{RDF}$ ) recorded maximum $\%$ of pore space $(57.22 \%)$ followed by $(56.1 \%)$ with $\mathrm{T}_{3}$ (PSB@20g kg $\mathrm{kg}^{-1}$ 0\%RDF and $\mathrm{T}_{2}$ (Azospirillum@20g kg-1 $+0 \% \mathrm{RDF}$ ) having (56.1\%). T 10 (Azospirillum + PSB@20 $\mathrm{g} \mathrm{kg}^{-1}+$ $75 \% \mathrm{RDF}$ ) recorded the minimum with $49.1 \%$.Overuse of fertilizers every year causes formation and concentration of mineral salts of fertilizers leading to compaction and soil degradation in the long-term (Massar et al., 2016). In some soils dissolution of salts due to irrigation causes dispersion of soil particles. Once aggregates are dispersed, fine clay particles leach into soil pores and block them. These fine and structure-less substances cover the soil surface which hinders water penetration and forms a hard and impermeable layer (Stawinski et al., 2011).

\section{Water retaining capacity $(\%)$}

Treatment $\mathrm{T}_{4}$ (Azospirillum+PSB@20 gm kg-1 $+0 \% \mathrm{RDF})$ recorded maximum Water retaining capacity $(62.1 \%)$ followed by (61.5\%) with $\mathrm{T}_{3}$ (PSB@ $\left.20 \mathrm{gm} \mathrm{kg}^{-1}+0 \% \mathrm{RDF}\right)$. $\mathrm{T}_{9}$ (PSB@20 gm kg-1 + 75\% RDF [N(112.5 $\left.\left.\mathrm{kg} \mathrm{ha}{ }^{-1}\right), \mathrm{P}\left(45 \mathrm{~kg} \mathrm{ha}^{-1}\right), \mathrm{K}\left(30 \mathrm{~kg} \mathrm{ha}^{-1}\right)\right]$ recorded the minimum $(56.12 \%)$.

Biofertilizers are basically microbes which can condition the soil structure by improving its physical properties. This might have led to an increase in no of soil pores and thereby an increase in water retaining capacity. Similar findings were observed by Amarjeet et al., (2014) (Table 2). 
Table.1 Pre sowing analysis of soil samples

\begin{tabular}{|c|c|}
\hline Parameters & Results \\
\hline Sand (\%) & 65.41 \\
\hline Silt (\%) & 21.00 \\
\hline Clay $(\%)$ & 13.59 \\
\hline Texture of soil & Sandy loam \\
\hline Bulk density $\left(\mathrm{Mg} \mathrm{m}^{-3}\right)$ & 1.26 \\
\hline Particle density $\left(\mathrm{Mg} \mathrm{m}^{-3}\right)$ & 2.50 \\
\hline Pore space $(\%)$ & 55.00 \\
\hline Water retaining capacity $(\%)$ & 61.96 \\
\hline Specific gravity & 2.29 \\
\hline Soil colour & Light yellowish brown \\
\hline Soil pH $(1: 2) w / v$ & 7.6 \\
\hline Electrical conductivity $\left(\mathrm{dS} \mathrm{m}^{-1}\right)$ & 0.16 \\
\hline Organic carbon $(\%)$ & 0.42 \\
\hline Available nitrogen $\left(\mathrm{kgha}^{-1}\right)$ & 240 \\
\hline Available phosphorous $\left(\mathrm{kgha}^{-1}\right)$ & 14.01 \\
\hline Available potassium $\left(\mathrm{kgha}^{-1}\right)$ & 107.20 \\
\hline
\end{tabular}

Table.2 Post harvest physical properties of soil

\begin{tabular}{|l|c|c|c|c|c|}
\hline Treatments & $\begin{array}{c}\text { Bulk } \\
\text { density } \\
\left(\mathbf{M g ~ m}^{-3}\right)\end{array}$ & $\begin{array}{c}\text { Particle } \\
\text { density } \\
\left(\mathbf{M g m}^{-3}\right)\end{array}$ & $\begin{array}{c}\text { Pore space } \\
\mathbf{( \% )}\end{array}$ & $\begin{array}{c}\text { Water } \\
\text { retaining } \\
\text { capacity } \mathbf{( \% )}\end{array}$ & $\begin{array}{c}\text { Specific } \\
\text { gravity }\end{array}$ \\
\hline $\mathbf{T}_{\mathbf{1}}$ & 1.11 & 2.5 & 55.55 & 60.1 & 2.1 \\
\hline $\mathbf{T}_{\mathbf{2}}$ & 1.1 & 2.47 & 56.1 & 61.1 & 2.12 \\
\hline $\mathbf{T}_{\mathbf{3}}$ & 1.12 & 2.72 & 56.1 & 61.5 & 2.15 \\
\hline $\mathbf{T}_{\mathbf{4}}$ & 1.04 & 2.44 & 57.2 & 62.1 & 2.19 \\
\hline $\mathbf{T}_{\mathbf{5}}$ & 1.17 & 2.53 & 52.3 & 58.9 & 2.26 \\
\hline $\mathbf{T}_{\mathbf{6}}$ & 1.19 & 2.61 & 52.7 & 58.4 & 2.29 \\
\hline $\mathbf{T}_{\mathbf{7}}$ & 1.16 & 2.58 & 51.8 & 57.2 & 2.35 \\
\hline $\mathbf{T}_{\mathbf{8}}$ & 1.23 & 2.96 & 50.5 & 56.8 & 2.42 \\
\hline $\mathbf{T}_{\mathbf{9}}$ & 1.22 & 2.87 & 50.5 & 56.1 & 2.42 \\
\hline $\mathbf{T}_{\mathbf{1 0}}$ & 1.23 & 2.92 & 49.1 & 56.2 & 2.45 \\
\hline F-Test & $\mathrm{NS}$ & $\mathrm{NS}$ & $\mathrm{S}$ & $\mathrm{S}$ & $\mathrm{S}$ \\
\hline S. Em. $\mathbf{( \pm )}$ & 0.06 & 0.10 & 0.94 & 0.58 & 0.11 \\
\hline C.D. at $\mathbf{0 . 5}$ & 0.19 & 0.29 & 2.78 & 1.71 & 0.34 \\
\hline
\end{tabular}


Table.3 Post harvest chemical analysis of soil

\begin{tabular}{|c|c|c|c|c|c|c|}
\hline Treatments & $\begin{array}{l}\text { Soil pH } \\
(1: 2) w / v\end{array}$ & $\begin{array}{c}\mathrm{EC} \\
\left(\mathrm{dSm}^{-3}\right)\end{array}$ & $\begin{array}{l}\text { O.C. } \\
(\%)\end{array}$ & $\begin{array}{c}\text { Available } \\
\text { Nitrogen }\left(\mathrm{kgha}^{-1}\right)\end{array}$ & $\begin{array}{c}\text { Available } \\
\text { phosphorous } \\
\left(\mathrm{kgha}^{-1}\right)\end{array}$ & $\begin{array}{c}\text { Available } \\
\text { Potassium } \\
\left(\text { kgha }^{-1}\right)\end{array}$ \\
\hline $\mathbf{T}_{1}$ & 7.3 & 0.06 & 0.42 & 206.8 & 16.1 & 113.4 \\
\hline $\mathbf{T}_{2}$ & 7.21 & 0.18 & 0.48 & 267.5 & 16.5 & 122.4 \\
\hline $\mathbf{T}_{3}$ & 7.23 & 0.21 & 0.49 & 251.4 & 16.7 & 119.4 \\
\hline $\mathbf{T}_{4}$ & 7.15 & 0.22 & 0.52 & 289.2 & 17.1 & 125.4 \\
\hline $\mathbf{T}_{5}$ & 7.41 & 0.24 & 0.58 & 292.1 & 27.6 & 173.5 \\
\hline $\mathbf{T}_{6}$ & 7.43 & 0.15 & 0.6 & 290.4 & 28.3 & 168.6 \\
\hline $\mathbf{T}_{7}$ & 7.35 & 0.25 & 0.62 & 298.6 & 28.7 & 172.4 \\
\hline $\mathbf{T}_{8}$ & 7.61 & 0.26 & 0.64 & 309.3 & 29.7 & 186.2 \\
\hline $\mathbf{T}_{9}$ & 7.57 & 0.26 & 0.66 & 304.3 & 31.5 & 182.3 \\
\hline $\mathbf{T}_{10}$ & 7.62 & 0.27 & 0.7 & 314.3 & 32.3 & 197.8 \\
\hline F-Test & S & $\mathrm{S}$ & $\mathrm{S}$ & S & $\mathrm{S}$ & S \\
\hline S.Em. ( \pm$)$ & 0.194 & 0.07 & 0.08 & 1.40 & 1.13 & 1.79 \\
\hline C.D. at 0.5 & 0.399 & 0.20 & 0.24 & 4.15 & 3.35 & 5.32 \\
\hline
\end{tabular}

\section{Specific gravity}

Treatment $\mathrm{T}_{10}$ (Azospirillum +PSB@20 gm $\mathrm{kg}^{-1}+75 \%$ RDF [N(112.5 $\left.\mathrm{kg} \mathrm{ha}^{-1}\right), \mathrm{P}\left(45 \mathrm{~kg} \mathrm{ha}^{-}\right.$ $\left.\left.{ }^{1}\right), \mathrm{K}\left(30 \mathrm{~kg} \mathrm{ha}^{-1}\right)\right]$ recorded maximum Specific gravity (2.45) followed by (2.42)with $\mathrm{T}_{9}$ (PSB@20 gm kg-1 $\left.\left.\mathrm{ha}^{-1}\right), \mathrm{P}\left(45 \mathrm{~kg} \mathrm{ha}^{-1}\right), \mathrm{K}\left(30 \mathrm{~kg} \mathrm{ha}^{-1}\right)\right]$ and $\mathrm{T}_{8}$ (Azospirillum @ $20 \mathrm{gm} \mathrm{kg}^{-1}+75 \% \mathrm{RDF}[$ $\mathrm{N}\left(112.5 \mathrm{~kg} \mathrm{ha}^{-1}\right), \mathrm{P}\left(45 \mathrm{~kg} \mathrm{ha}^{-1}\right), \mathrm{K}(30 \mathrm{~kg}$ $\left.\left.\mathrm{ha}^{-1}\right)\right] . \mathrm{T}_{1}$ recorded the minimum $(2.1)$.

\section{Chemical properties}

\section{Organic carbon $(\%)$}

Treatment $\mathrm{T}_{10}$ (Azospirillum +PSB@20 gm $\mathrm{kg}^{-1}+75 \%$ RDF $\left[\mathrm{N}\left(112.5 \mathrm{~kg} \mathrm{ha}^{-1}\right), \quad \mathrm{P}(45\right.$ $\left.\left.\left.\mathrm{kg} \mathrm{ha}^{-1}\right), \mathrm{K}\left(30 \mathrm{~kg} \mathrm{ha}^{-1}\right)\right]\right)$ recorded maximum Organic carbon (\%) $(0.76 \%)$ which was statistically at par with $(0.66 \%)$ with $\mathrm{T}_{9}$ (Azospirillum + PSB@20 gm kg-1 + 75\% RDF [N(112.5 kg ha $\left.{ }^{-1}\right), \quad \mathrm{P}\left(45 \mathrm{~kg} \mathrm{ha}{ }^{-1}\right), \quad \mathrm{K}(30$ $\left.\left.\mathrm{kg} \mathrm{ha}^{-1}\right)\right] . \mathrm{T}_{1}$ (control) recorded the minimum $(0.42 \%)$. Higher values of residual soil fertility with the application of high dose of
RDF, applied with or without biofertilizer (s), might be due to the fact that application of chemical fertilizers leads to better root development, accordingly higher soil organic carbon (SOC), and consequently increases the levels of many soil nutrients Nambiar et al., (1992). Any increase in the organic carbon content also will result in increased nutrient availability to the plant by augmenting soil biological activity, thereby enhancing nutrient use efficiency Ramalakshmi et al., (2008). The results obtained are in agreement with those attained by Sharma and Banik in (2014) (Table 3).

\section{$\mathrm{EC}\left(\mathrm{dSm}^{-1}\right)$}

Treatment $\mathrm{T}_{10}$ (Azospirillum + PSB@20 gm $\mathrm{kg}^{-1}+75 \%$ RDF [ N(112.5 kg ha $\left.{ }^{-1}\right), \mathrm{P}(45 \mathrm{~kg}$ $\left.\left.\mathrm{ha}^{-1}\right), \mathrm{K}\left(30 \mathrm{~kg} \mathrm{ha}{ }^{-1}\right)\right]$ recorded maximum Electrical conductivity $\left(0.27 \mathrm{dSm}^{-1}\right)$ which was followed by $(0.26 \%)$ with $\mathrm{T}_{9}$ (PSB@20 $\mathrm{gm} \mathrm{kg}^{-1}+75 \%$ RDF [N(112.5 $\left.\mathrm{kg} \mathrm{ha}^{-1}\right), \mathrm{P}(45 \mathrm{~kg}$ $\left.\left.\mathrm{ha}^{-1}\right), \mathrm{K}\left(30 \mathrm{~kg} \mathrm{ha}^{-1}\right)\right]$ and $\mathrm{T}_{8}$ (Azospirillum @ $20 \mathrm{gm} \mathrm{kg}^{-1}+75 \% \mathrm{RDF}\left[\mathrm{N}\left(112.5 \mathrm{~kg} \mathrm{ha}^{-1}\right) . \mathrm{T}_{1}\right.$ (control) recorded the minimum $\left(0.06 \mathrm{dSm}^{-1}\right)$. 
Different Researchers noted notable changes in soil reaction values and EC with the quantity and nature of the chemical fertilizers used. The magnitude of these changes in soil properties is largely determined by the amount and nature of $\mathrm{C}$ compounds released, as well as intrinsic soil characteristics. Similar results were obtained by Banik and Sharma in (2014).

pH (1:2) w/v

Treatment $\mathrm{T}_{10}$ (Azospirillum +PSB@20 gm $\mathrm{kg}^{-1}+75 \% \mathrm{RDF}\left[\mathrm{N}\left(112.5 \mathrm{~kg} \mathrm{ha}{ }^{-1}\right), \quad \mathrm{P}(45\right.$ $\left.\left.\mathrm{kg} \mathrm{ha}^{-1}\right), \mathrm{K}\left(30 \mathrm{~kg} \mathrm{ha}^{-1}\right)\right]$ recorded maximum $\mathrm{pH}$ (7.62) which was followed by (7.61)with $\mathrm{T}_{8}$ (Azospirillum @ $20 \mathrm{gm} \mathrm{kg}^{-1}+75 \% \mathrm{RDF}[$ $\left.\mathrm{N}\left(112.5 \mathrm{~kg} \mathrm{ha}^{-1}\right), \mathrm{P}\left(45 \mathrm{~kg} \mathrm{ha}^{-1}\right), \mathrm{K}\left(30 \mathrm{~kg} \mathrm{ha}^{-1}\right)\right]$ and $\mathrm{T}_{9}$ (PSB @20 gm kg-1 $+75 \% \mathrm{RDF}[$ $\mathrm{N}\left(112.5 \mathrm{~kg} \mathrm{ha}^{-1}\right) . \mathrm{T}_{4}$ (Azospirillum + PSB@20 $\mathrm{gm} \mathrm{kg}^{-1}+0 \% \mathrm{RDF}$ ) recorded the minimum $\mathrm{pH}$ ) (7.15). There was only a slight variation in the $\mathrm{pH}$ due to biofertilizer application and crop growth. The biofertilizer inoculation resulted in the shift in the $\mathrm{pH}$ reducing the alkalinity slightly. Illmer and Schinner (1995) also proposed that acidification of rhizosphere could be through liberation of organic acids by proton extrusion mechanism. The results are in agreement with Ramalakshmi et al., (2008).

\section{Available Nitrogen $\left(\mathrm{kg} \mathrm{ha}^{-1}\right)$}

Treatment $\mathrm{T}_{10}$ (Azospirillum + PSB@20 gm $\mathrm{kg}^{-1}+75 \% \mathrm{RDF}\left[\mathrm{N}\left(112.5 \mathrm{~kg} \mathrm{ha}^{-1}\right), \mathrm{P}(45 \mathrm{~kg}\right.$ $\left.\left.\mathrm{ha}^{-1}\right), \mathrm{K}\left(30 \mathrm{~kg} \mathrm{ha}{ }^{-1}\right)\right]$ recorded maximum Available Nitrogen $\left(314.3 \mathrm{~kg} \mathrm{ha}^{-1}\right)$ which was followed by $\left(309.3 \mathrm{~kg} \quad \mathrm{ha}^{-1}\right)$ with $\mathrm{T}_{8}$ (Azospirillum @20 gm kg-1 $+75 \% \mathrm{RDF}$ $\left.\mathrm{N}\left(112.5 \mathrm{~kg} \mathrm{ha}^{-1}\right), \mathrm{P}\left(45 \mathrm{~kg} \mathrm{ha}^{-1}\right), \mathrm{K}\left(30 \mathrm{~kg} \mathrm{ha}^{-1}\right)\right]$ and $\mathrm{T}_{9}$ (PSB @20 gm kg $\mathrm{gm}^{-1}+75 \% \mathrm{RDF}[$ $\mathrm{N}\left(112.5 \mathrm{~kg} \mathrm{ha}^{-1}\right)$, having (304.3 kg ha $\left.{ }^{-1}\right) . \mathrm{T}_{1}$ (control) recorded the minimum with (206.8 $\mathrm{kg} \mathrm{ha}^{-1}$ ). Azospirillum in maize crop enhance the crop yield in the range similar to $60 \mathrm{~kg}$ urea N/ha Smith et al., (1996). Mertens and Hess (1984) reported that inoculation of Azospirillum in wheat increased available nitrogen in soil by fixation of atmospheric nitrogen. Thereby, the use of both the inoculants (Azospirillum + PSB) increases nutrient use efficiency and improve the soil properties. Huge amounts of soil organic nutrients, which are otherwise unavailable to plants directly, can be made accessible only after their mineralization, a process mediated by Biofertilizers. Similar results were obtained by Sharma and Banik (2014) and Ramalakshmi (2008).

\section{Phosphorus (kg ha $\left.{ }^{-1}\right)$}

Treatment $\mathrm{T}_{10}$ (Azospirillum +PSB@20 gm $\mathrm{kg}^{-1}+75 \%$ RDF[ N(112.5 kg ha $\left.{ }^{-1}\right), \mathrm{P}(45 \mathrm{~kg}$ $\left.\left.\mathrm{ha}^{-1}\right), \mathrm{K}\left(30 \mathrm{~kg} \mathrm{ha}^{-1}\right)\right]$ recorded maximum available Phosphorous (32.3 kg ha ${ }^{-1}$ ) which was followed by $\left(31.5 \mathrm{~kg} \mathrm{ha}^{-1}\right)$ with $\mathrm{T}_{9}$ (PSB @20 $\mathrm{gm} \mathrm{kg}^{-1}+75 \% \mathrm{RDF}$ [N(112.5 kg ha-1), $\left.\mathrm{P}\left(45 \quad \mathrm{~kg} \mathrm{ha}{ }^{-1}\right), \quad \mathrm{K}\left(30 \mathrm{~kg} \mathrm{ha}^{-1}\right)\right]$ and $\mathrm{T}_{8}$ (Azospirillum @20 gm kg-1 +75\%RDF[ $\mathrm{N}\left(112.5 \mathrm{~kg} \mathrm{ha}^{-1}\right)$, having (29.7 $\left.\mathrm{kg} \mathrm{ha}^{-1}\right) . \mathrm{T}_{1}$ (control) recorded the minimum with $(16.1 \mathrm{~kg}$ $\left.\mathrm{ha}^{-1}\right)$.

Phosphate solubilizing bacterium/ microorganism has enormous potential to solubilize about 50 to 60 per cent of fixed phosphorus in the soil by secreting organic acids with in a short time. The increased availability of soil phosphorus due to phosphobacteria inoculation was observed by Sundaravadivel et al., (1999). Thereby, the use of both the inoculants (Azospirillum + PSB) increases nutrient use efficiency and improve the soil properties. Huge amounts of soil organic nutrients, which are otherwise unavailable to plants directly, can be made accessible only after their mineralization, a process mediated by Biofertilizers. Similar results were obtained by Sharma and Banik (2014) and Ramalakshmi (2008). 


\section{Potassium $\left(\mathrm{kg} \mathrm{ha}^{-1}\right)$}

Treatment $\mathrm{T}_{10}$ (Azospirillum +PSB@20 gm $\mathrm{kg}^{-1}+75 \%$ RDF [ N(112.5 $\left.\mathrm{kg} \mathrm{ha}^{-1}\right), \mathrm{P}(45 \mathrm{~kg}$ $\left.\left.\mathrm{ha}^{-1}\right), \mathrm{K}\left(30 \mathrm{~kg} \mathrm{ha}{ }^{-1}\right)\right]$ recorded maximum Available Potassium $\left(197.8 \mathrm{~kg} \mathrm{ha}^{-1}\right)$ which was followed by $\left(186.2 \mathrm{~kg} \mathrm{ha}^{-1}\right)$ with $\mathrm{T}_{8}$ (Azospirillum @20 $\mathrm{gm} \mathrm{kg}^{-1}+75 \% \mathrm{RDF}$ [N(112.5 $\left.\mathrm{kg} \mathrm{ha}^{-1}\right), \mathrm{P}\left(45 \mathrm{~kg} \mathrm{ha}^{-1}\right), \mathrm{K}(30 \mathrm{~kg} \mathrm{ha}$ $\left.{ }^{1}\right)$ ] and $\mathrm{T}_{9}$ (PSB @20 gm kg${ }^{-1}+75 \% \mathrm{RDF}[$ $\mathrm{N}\left(112.5 \mathrm{~kg} \mathrm{ha}^{-1}\right)$, having $\left(182.3 \mathrm{~kg} \mathrm{ha}^{-1}\right) . \mathrm{T}_{1}$ (control) recorded the minimum with (206.8 $\left.\mathrm{kg} \mathrm{ha}{ }^{-1}\right)$. Synergistic interactions are well known for $\mathrm{N} \times \mathrm{K}$ and $\mathrm{N} \times \mathrm{P}$ interactions Aulakh and Malhi (2005). They are not only important for the yield but also help to explain their combined effect on root growth, and the relevance for synchronized applications of, for example, $\mathrm{N}$ and $\mathrm{K}$, during the growing season Aulakh and Malhi (2005), ultimately resulting in improved nutrient use efficiency (NUE).

The soils of the biofertilizer inoculated treatment had a higher content of available nitrogen, phosphorus and potassium. These results showed that there is a direct relationship between inoculation and the available nutrients. Similar results were obtained by Sharma and Banik (2014) and Ramalakshmi et al., (2008).

In conclusion the physical properties do not change much in a single cropping season as they are intrinsic properties of soil. Treatment $\mathrm{T}_{10}$ (Azospirillum + PSB@20 $\mathrm{gm} \mathrm{kg}^{-1}+75 \%$ RDF [N(112.5 $\left.\mathrm{kg} \mathrm{ha}^{-1}\right), \mathrm{P}\left(45 \mathrm{~kg} \mathrm{ha}^{-1}\right), \mathrm{K}(30$ $\left.\left.\mathrm{kg} \mathrm{ha}{ }^{-1}\right)\right]$ recorded maximum Available nitrogen, phosphorous and potassium and organic carbon. Baby corn being a heavy feeder crop showed optimum results with a $25 \%$ less RDF. Co inoculation of biofertilizers have increased the soil fertility levels considerably than the control plots making native nutrients available to crop plants and improving soil health.

\section{References}

Pandey, A.K. and Prakesh, V. and Mani, V.P. and Singh, Rashmi, (2000). Effect of rate of nitrogen and time of application on yield and economics of babycorn (Zea mays L.) Indian Journal of Agronomy 45(06): 338-343.

Rai, S., \& Gaur, A. (1988). Characterization of Azotobacter spp. and effect of Azotobacter and Azospirillum as inoculant on the yield and N-uptake of wheat crop. Plant and Soil, 109(1), 131-134.

Datta, M, Banik, S. and Laskar, S. (1992). Effect of inoculation of phosphate dissolving bacteria on rice (Oryza sativa) in acid soils. Indian J. Agric. Res., 62(7): 482- 485

Khalid A, M.S. Khan, Azeem Khalid, Muhammad Arshad, Baby Shaharoona, and Tariq Mahmood (2009), Microbial Strategies for Crop Improvement Springer-Verlag Berlin Heidelberg, DOI: 10.1007/978-3-642-01979-1_7

Ramalakshmi A, M. Iniyakumar and S. Anthoniraj (2018). Influence of biofertilizers on soil physico-chemical and biological properties during cropping period Asian Journal of Bio Science, Vol. 3 No. 2 : 348-351

Ravi Chandra Sharma and PabitraBanik (2014) Vermicompost and Fertilizer Application: Effect on Productivity and Profitability of Baby Corn (Zea mays L.) and Soil Health Compost Science \& Utilization- Taylor \& Francis Group, 22: 83-92,

Subbiah, B.V. and Asija, G.L. 1956. A rapid procedure for the determination of available nitrogen in soils. Curr. Sci., 25(7): 259-260.

Walkley and Black. (1947) Estimation of soil organic carbon by the chromic acid titration method. Soil Sci., 37: 29-38.

Aulakh, M.S., Malhi, S.S. (2005). Interactions 
of nitrogen with other nutrients and water: effect on crop yield and quality, nutrient use efficiency, carbon sequestration, and environmental pollution. Adv. Agron. 86, 341-409.

Sundaravadivel, K., Chitdeswari, T., Subramanian, S. and Krishnadoss,D. (1999). Cost effective phosphorus practices for rainfed cotton in vertisols. Madras Agricultural Journal, 86(7-9): 384-388.

Olsen, S.R., Cole, C.V., Watanabe, F.S. and
Dean, L.A. (1954). Estimation of available phosphorus in soils by extraction with sodium bicarbonate. U.S. Dept. Agrl. Circ. 939, Washington, D.C, Pp. 24.

Illmer, P. and Schinner, F. (1995). Solubilization of inorganic calcium phosphates - $\quad$ solubilization mechanisms. Soil Biology and Biochemistry, 27(3): 257-263.

\section{How to cite this article:}

Cici Maria Antony, Narendra Swaroop, Tarence Thomas, Arun A. David and Smriti Rao, P. 2020. Effect of Different Levels of Inorganics and Biofertilizers on Post Harvest Soil PhysicoChemical Properties of Baby Corn (Zea mays L.) Variety- G-5414. Int.J.Curr.Microbiol.App.Sci. 9(09): 1501-1508. doi: https://doi.org/10.20546/ijcmas.2020.909.190 